\title{
O NOvo Regulamento Sanitário INTERNACional
}

\author{
THE NEW INTERNATIONAL HEALTH REGULATIONS
}

\author{
Carolina B. Gomes* \\ Larissa de P. Gonzaga e Castro**
}

\section{RESUMO}

A mais recente revisão do Regulamento Sanitário Internacional (RSI), publicada em 2005, trouxe inovações com o intuito de transpor as limitações enfrentadas pelo regulamento de 1969 , que se tornaram cada vez mais aparentes com o ressurgimento de antigas doenças e o aparecimento de novas, nas décadas de 1970 e 1980. Por meio da revisão da literatura, dos regulamentos sanitários internacionais $(1951,1969,2005)$ e de sítios eletrônicos de entidades de saúde, foi feito um estudo essencialmente bibliográfico e documental, com o objetivo de identificar e apresentar as ferramentas trazidas pelo novo RSI. Apesar de esse instrumento se mostrar promissor para auxiliar na resposta mundial a um surto, há outros fatores envolvidos, como as diferenças econômicas, políticas, culturais e religiosas entre os Estados-Membros da OMS; é preciso considerar que alguns países não contam com o mínimo de infraestrutura básica para atender satisfatoriamente situações menos complexas que a transnacionalização dos riscos, e possivelmente jamais conseguirão atender às capacidades nacionais básicas para detectar, avaliar, notificar e informar uma Emergência de Saúde Pública de Importância Internacional (ESPII) sem ajuda técnica e financeira, não prevista expressamente pelo novo instrumento regulatório. Além disso, a visão de que se podem controlar totalmente a ocorrência e o alastramento de eventos em saúde é demasiadamente otimista. Sempre que possível, o intuito do regulamento é evitar a ocorrência de eventos em saúde; porém, quando isso

$\left(^{*}\right)$ Especialista em Atenção Farmacêutica e Vigilância Sanitária, Pontifícia Universidade Católica de Goiás (PUC/GO); especialista em Farmacologia, Universidade Federal de Lavras (UFLA). Brasília/ DF - Brasil. E-mail: carol.gomes@gmail.com.

${ }^{* *}$ ) Mestre em Direito Internacional, Universidad de Sevilla (Espanha); Especialista em Saúde Global, Escola Nacional de Saúde Pública - Fundação Oswaldo Cruz (ENSP/FIOCRUZ). Professora de graduação e pós-graduação na Universidade do Distrito Federal, Fiocruz e IFAR. Brasília/DF - Brasil. Consultora jurídica. E-mail: prof.larissa@gmail.com.

Texto recebido em 06.12.2011. Revisado em 14.09.2012. Aprovado em 20.09.2012 
não acontece, o objetivo torna-se minimizar o alastramento e diminuir a gravidade das consequências.

\title{
Palavras-chave:
}

Cadeia de Comunicação; Emergência de Saúde Pública de Importância Internacional (ESPII); Regulamento Sanitário Internacional (RSI);

\begin{abstract}
The most recent revision of the International Health Regulations (IHR), published in 2005, brought innovations with the purpose of overcoming the limitations faced by the 1969 regulations, which became more and more evident with the reoccurrence of old diseases and the appearance of new ones on the 70s and $80 \mathrm{~s}$. Through the review of the literature, of the international health regulations $(1951,1969,2005)$ and of healthcare organizations websites, an essentially bibliographic and documental study was performed, with the objective of identifying and presenting the tools brought by the new RSI. Although this instrument shows promise in assisting in the global response to an outbreak, there are other factors involved, such as the economical, political, cultural and religious differences among the WHO Member-States: one must consider that some countries do not have the minimum basic infrastructure to satisfactorily meet less complex situations than the transnationalization of risks, and possibly will never be able to meet the basic national capacities to detect, assess, notify and inform a Public Health Emergency of International Importance (PHEIC) without the technical and financial assistance, not expressly foreseen by the new regulatory instrument. Moreover, the view that one can fully control the occurrence and spread of health events is greatly optimistic. Whenever possible, the intent of the regulations is to prevent the occurrence of health events, but when it is not possible, the goal becomes to minimize the spread and mitigate the severity of the consequences.
\end{abstract}

\section{Keywords:}

Communication Chain; Public Health Emergency of International Concern (PHEIC); International Health Regulations (IHR).

\section{INTRODUÇÃO}

A globalização das doenças, entendida como sua difusão mundial, teve início no final do século XV, com a descoberta das Américas ${ }^{(1)}$. Entretanto, desde

(1) BERLINGUER, Giovanni. Globalização e saúde global. Estudos Avançados, São Paulo, v. 13, n. 35, p. 21-38, 1999. 
os primórdios da civilização o instinto de autopreservação induz as pessoas a adotarem medidas com o intuito de se protegerem contra as ameaças representadas por outros povos, incluídas aí aquelas relacionadas a seu bem-estar físico. Embora o termo "quarentena" date do final do século XIV, tanto a Bíblia quanto o Alcorão já fazem referência ao isolamento de leprosos e, no século VII, a primeira medida transnacional de caráter sanitário pode ser observada, quando a China instituiu uma política para evitar a entrada em seu território de marinheiros com peste ${ }^{(2)}$.

A difusão mundial de doenças transmissíveis levou a uma unificação microbiana a partir do século XVI, mas somente três séculos mais tarde, com o aumento da velocidade de disseminação dos riscos à saúde e sua capacidade de cruzar fronteiras, percebeu-se que a contenção da disseminação mundial precisava ocorrer em cooperação com os países vizinhos para que fosse efetiva - "ganhamos todos ou perdemos todos"(3) -, criando-se assim uma crescente demanda por um regulamento sanitário capaz de evitar, ou ao menos minimizar, 0 alastramento internacional de doenças.

A primeira regulamentação internacional com intuito de impedir a disseminação através das fronteiras dos países surgiu na Europa em meados do século XIX. Em 1851 ocorreu a Primeira Conferência Sanitária Internacional em Paris, seguida das conferências de 1892 e 1897 para negociação de medidas para contenção da cólera e da praga, respectivamente.

O Primeiro Regulamento Sanitário Internacional, acordo internacional juridicamente vinculante, foi adotado durante a $4^{\mathrm{a}}$ Assembleia Mundial da Saúde, em 1951, três anos após a entrada em vigor da constituição da Organização Mundial da Saúde (OMS) ${ }^{(4)}$. É considerado o primeiro código internacional de medidas para prevenir a propagação de determinadas enfermidades infecciosas (cólera, peste, febre amarela, varíola, febre recorrente e tifo) e de requisitos sobre notificação de casos e apresentação de informes, assim como a adoção de medidas sanitárias nos pontos de entrada e saída de pessoas e mercadorias nos países.

Durante a $22^{\mathrm{a}}$ Assembleia Mundial da Saúde, no ano de 1969, foi adotado o novo Regulamento Sanitário Internacional, dispondo sobre a contenção e notificação de casos de cólera, peste e febre amarela. Em 1973 foram

(2) ORGANIZACIÓN MUNDIAL DE LA SALUD. Evolución de la seguridad sanitaria. In: INFORME sobre la salud en el mundo 2007 - Un porvenir más seguro: protección de la salud pública mundial en el siglo XXI. Geneva, 2007. p. 1-15. Disponível em: <http://www.who.int/whr/2007/07_report_es.pdf>. Acesso em: 13 dez. 2009.

(3) GONZALO, Miguel Mínguez. The new international health regulations (2005 IHR). Revista Española de Salud Pública, Madrid, v. 81, n. 3, p. 239-246, maio/jun. 2007.

(4) ORGANIZAÇÃO MUNDIAL DA SAÚDE. Constitución de la Organización Mundial de la Salud. Disponível em: <http://www.who.int/governance/eb/who_constitution_sp.pdf>. Acesso em: 14 ago. 2012. 
modificados itens relativos à cólera, quando o Certificado de Vacinação contra a doença deixou de ser obrigatório e em 1981 se excluiu a varíola, devido a sua erradicação(5).

O Regulamento Sanitário Internacional de 1969 apresentava várias limitações, não atendendo de forma adequada aos objetivos a que se propunha, e vinha sofrendo inúmeras censuras principalmente nas últimas décadas. Seu aspecto mais criticado era sua limitação de cobertura, pois regulava taxativamente a cólera, a peste e a febre amarela, mostrando-se ineficaz para enfrentar as modificações ocorridas na situação epidemiológica mundial(6).

Após a revisão do RSI em 1969, suas limitações tornaram-se cada vez mais aparentes, com o ressurgimento de doenças antigas, como a cólera, e com o aparecimento de novas doenças, como o ebola e o HIVIAIDS nas décadas de 1970 e 1980. Entretanto, foram as epidemias de Síndrome Respiratória Aguda Grave (SARS) em 2002 e de Influenza Aviária (H5N1) em 2003 que impulsionaram a finalização de sua mais recente revisão, que estava sendo feita desde 1995. Após 36 anos da entrada em vigor da revisão de 1969 do RSI, foi publicado o RSI 2005, que passou a valer em 15 de junho de 2007 e cujo prazo de cinco anos para adequação pelos Estados-Membros da OMS termina agora em $2012^{(7)}$.

Os Estados-Membros podem requerer que esse prazo seja prorrogado por dois anos, mediante justificativa técnica e apresentação de um plano de implementação. Podem ainda, em circunstâncias excepcionais, apresentar um novo plano de implementação e solicitar ao diretor-geral da OMS outra prorrogação de no máximo dois anos. Dessa forma, todos os Estados-Membros deverão obrigatoriamente ter se adequado aos requisitos do novo RSI até, no máximo, $2016^{(8)}$.

Apesar de ainda restarem alguns anos até o término desse prazo, a recente epidemia de Influenza A (H1N1) demonstrou a importância do RSI 2005 e como ele possibilitou uma resposta melhor e mais rápida perante esse evento, em comparação com aquela observada para a epidemia de SARS. O objetivo do regulamento de facilitar a comunicação e a formulação de um plano de ação após a identificação da Emergência de Saúde Pública de Importância Internacional,

(5) WORLD HEALTH ORGANIZATION. International Health Regulations (1969). Disponível em: <http://www.who.int/csr/ihr/ihr1969.pdf>. Acesso em 14 ago. 2012. Regulamento Sanitário Internacional (1969).

(6) FIDLER, David P. Globalization, international law, and emerging infectious diseases. Emerging Infectious Diseases, v. 2, n. 2, 1996.

(7) SUíçA. Artigo 5 do Regulamento Sanitário Internacional de 2005. Altera a antiga versão do regulamento. WORLD HEALTH ORGANIZATION. International Health Regulations (2005). 2. ed. 2008. Disponível em: <http://whqlibdoc.who.int/publications/2008/9789241580410_eng.pdf>. Acesso em: 13 dez. 2009.

(8) Id. Ibid. 
adiante denominada ESPII, foi atingido e, se não por outros motivos, este já faz do novo regulamento um sucesso ${ }^{(9)}$.

O objetivo deste trabalho foi identificar e apresentar as ferramentas trazidas pelo novo RSI, com intuito de superar as limitações do RSI de 1969: previsão de novas fontes de informações sobre ESPII e a possibilidade de desenvolvimento de um sistema internacionalizado de vigilância em saúde; ampliação dos eventos abrangidos; estruturação do sistema de identificação e notificação de eventos.

De forma a atingir esse objetivo, foi feito um estudo essencialmente bibliográfico e documental, por meio de uma revisão sistemática de trabalhos publicados em revistas científicas e da leitura e análise das versões dos regulamentos, em particular daquelas publicadas em 1969 e 2005.

Além disso, foram coletadas também informações disponíveis nos sítios eletrônicos de entidades de saúde, tais como da Organização Mundial da Saúde, da Organização Pan-Americana da Saúde, do Ministério da Saúde, da Agência Nacional de Vigilância Sanitária, do Global Public Health Intelligence Network (Gphin), do Program for Monitoring Emerging Diseases (ProMed-mail), do Global Disease Alert Map (HealthMap) e do Global Outbreak Alert and Response Network (Goarn), entre outros.

\section{AS NOVAS FONTES DE INFORMAÇÕES SOBRE ESPII PREVISTAS PELO RSI 2005 E A POSSIBILIDADE DE DESENVOLVIMENTO DE UM SISTEMA INTERNACIONALIZADO DE VIGILÂNCIA EM SAÚDE}

Uma das principais mudanças trazidas pelo novo RSI foi a possibilidade de uso de fontes não oficiais para coleta de informações. De acordo com o RSI 1969, a OMS somente poderia responder a uma epidemia mediante comunicação oficial voluntária do Estado-Membro. Por sua vez, o RSI 2005 permite à OMS ampliar as fontes além das notificações ou consultas, de forma a identificar alarmes e iniciar um processo de investigação em países que não tenham feito a notificação voluntária de eventos significativos ${ }^{(10)}$.

Essa ampliação da possibilidade de fontes de informação levou à internacionalização do sistema de vigilância em saúde, uma vez que a ação da OMS não depende somente da comunicação oficial voluntária do Estado-Membro; isso coloca os países em uma posição na qual eles não podem ignorar ou esconder

(9) KATZ, Rebecca. Use of Revised International Health Regulations During Influenza A (H1N1) Epidemic. Emerging Infectious Diseases, Atlanta GA, v. 15, n. 8, p. 1165-1170, ago. 2009.

(10) MERIANOS, Angela; PEIRIS, Malik. International Health Regulations. The Lancet, New York NY, v. 366, n. 9493, p. 1249-1251, Oct. 2005. 
informações sobre surtos que ocorrem em seu território(11). Vários sistemas de vigilância existentes e mantidos por países ou mesmo por entidades particulares contribuem para a coleta pela OMS de informações pertinentes à identificação de uma ESPII.

O Gphin, por exemplo, é um sistema mantido pelo governo canadense que contínua e sistematicamente coleta dados da internet sobre eventos de saúde em sete idiomas (inglês, francês, espanhol, russo, árabe, chinês simplificado e tradicional), por meio de palavras e frases-chave. As informações coletadas são automaticamente separadas por relevância e analisadas pelos funcionários da agência canadense, para então serem disponibilizadas para os usuários. Notificações de eventos que possam ocasionar consequências graves são enviadas imediatamente aos usuários ${ }^{(12)}$.

Da mesma forma, o ProMed-mail é um sistema mantido pela International Society for Infectious Diseases (ISID), dedicado à disseminação global de informações sobre surtos de doenças infecciosas e exposições agudas a toxinas que afetam a saúde humana, incluindo aquelas relacionadas a animais e plantas destinadas ao consumo humano e animal ${ }^{(13)}$.

Semelhante, o HealthMap é um sistema criado por Clark Freifeld e John Brownstein que reúne dados de diferentes fontes para obter uma visão abrangente e unificada do atual estado mundial das doenças infecciosas e seus efeitos sobre a saúde humana e animal(14). Por meio de um sistema automatizado de processamento de texto, os dados são agregados por doença e exibidos por local, facilitando o acesso ao alerta original, fornecendo assim um ponto de partida para a informação em tempo real sobre doenças infecciosas emergentes e com especial interesse para pessoas que trabalham com saúde pública, bem como para viajantes internacionais ${ }^{(15)}$.

Por sua vez, o Goarn é um sistema de colaboração técnica, mantido pela OMS com instituições e redes existentes que concentram seus recursos

(11) HEYMANN, David L. SARS and emerging infectious diseases: a challenge to place global solidarity above national sovereignty. Annals Academy of Medicine, Singapura, v. 35, n. 5, p. 350-353, 2006.

(12) PUBLIC HEALTH AGENCY OF CANADA. The Global Public Health Intelligence Network (GPHIN), Set. 2004. Disponível em: <http://www.phac-aspc.gc.ca/media/nr-rp/2004/2004_gphin-rmispbk-eng. php>. Acesso em: $13 \mathrm{dez}$. 2009. Apresenta dados sobre o sistema de vigilância sanitária internacional. (13) INTERNATIONAL SOCIETY FOR INFECTIOUS DISEASES. Program for Monitoring Emerging Diseases - ProMed-mail. 1986. Disponível em: <http://www.promedmail.org/pls/otn/f?p=2400:1950:2 415474060216450::NO:::>. Acesso em: 13 dez. 2009. Apresenta dados sobre o sistema de vigilância sanitária internacional.

(14) HEALTHMAP, 2007. Global Disease Alert Map. Disponível em: <http://www.healthmap.org/about. php>. Acesso em: $13 \mathrm{dez}$. 2009. Apresenta dados sobre o sistema de vigilância sanitária internacional. (15) BROWNSTEIN, John S.; FREIFELD, Clark C. HealthMap: the development of automated realtime internet surveillance for epidemic intelligence. Eurosurveillance, Suíça, v. 12, n. 48, pii=3322, Nov. 2007. Disponível em: <http://www.eurosurveillance.org/ViewArticle.aspx?Articleld=3322>. Acesso em: 13 dez. 2009. 
humanos e técnicos para uma rápida identificação, confirmação e resposta a surtos de importância internacional. O sistema fornece um quadro operacional para fazer a ligação entre esse conhecimento e a habilidade, de forma a manter a comunidade internacional em alerta para a ameaça de surtos e em constante prontidão para responder a ela(16).

\section{A AMPLIAÇÃO DOS EVENTOS ABRANGIDOS PELO RSI 2005}

De acordo com a OMS, "saúde é um estado de completo bem-estar físico, mental e social e não meramente a ausência de doenças ou enfermidades"(17). Por sua vez, o RSI 2005 define doença como "um adoecimento ou condição médica, independentemente da origem ou fonte, que causa ou poderia causar dano a humanos"(18). Em consonância com essas duas definições, uma importante mudança trazida pelo RSI 2005 foi a ampliação dos tipos de eventos abrangidos pelo regulamento. Diferentemente do RSI 1969, que restringia a notificação à OMS somente aos casos das doenças pré-definidas pelo regulamento (peste, cólera e febre amarela) ${ }^{(19)}$, o RSI 2005 vai além, com a criação do conceito de ESPII (20).

O novo regulamento define ESPII como "um evento extraordinário que constitua um risco para a saúde pública de outro Estado pela disseminação internacional da doença ou potencialmente e que exija uma resposta internacional coordenada"(21) e determina que cada Estado-Membro deverá analisar os eventos ocorridos em seu território com auxílio do Instrumento de Decisão,

(16) WORLD HEALTH ORGANIZATION. Global Outbreak Alert and Response Network - GOARN. Apr. 1948. Disponível em: <http://www.who.int/csr/outbreaknetwork/en>. Acesso em: 13 dez. 2009. Apresenta dados sobre o sistema de vigilância sanitária internacional.

(17) Organização Mundial da Saúde, abr. 1948. Definição de saúde dada pelo Preâmbulo da Constituição da Organização Mundial da Saúde adotada pela Conferência de Saúde Internacional, Nova York, 19-22 Junho, 1946; assinada em 22 de Julho de 1946 pelos representantes de 61 Estados (Registros Oficiais da Organização Mundial da Saúde, no. 2, p. 100) e vigorando a partir de 7 de Abril de 1948. WORLD HEALTH ORGANIZATION. WHO definition of Health. Apr. 1948. Disponivel em: <http://www.who.int/about/definition/en/print.html>. Acesso em: 13 dez. 2009.

(18) SUÍÇA. Artigo 1.1 do Regulamento Sanitário Internacional de 2005. Altera a antiga versão do regulamento. WORLD HEALTH ORGANIZATION. International Health Regulations (2005). 2. ed. 2008, cit.

(19) GENEVA. Parte V do Regulamento Sanitário Internacional de 1969. Altera a antiga versão do regulamento. WORLD HEALTH ORGANIZATION. International Health Regulations (1969). 3. ed. Disponível em: <http://www.who.int/csr/ihr/ihr1969.pdf>. Acesso em: 13 dez. 2009.

(20) RODIER, Guénaël; HARDIMAN, Max; PLOTKIN, B.; GANTER, B. Implementing the International Health Regulations (2005). Europe Eurosurveillance, Suíça, v. 11, n. 12, pii=664, Dec. 2006. Disponível em: <http://www.eurosurveillance.org/ViewArticle.aspx?Articleld=664>. Acesso em: 13 dez. 2009.

(21) SUÍÇA. Artigo 1.1 do Regulamento Sanitário Internacional de 2005. Altera a antiga versão do regulamento. WORLD HEALTH ORGANIZATION. International Health Regulations (2005). 2. ed. 2008, cit. 
constante no Anexo II do Regulamento, e notificar à OMS quando a avaliação assim concluir necessário ${ }^{(22)}$. Além de analisar os eventos ocorridos em seu próprio território, o RSI 2005 também imputa aos Estados-Membros, na medida do possível, a responsabilidade de notificar eventos ocorridos fora de seu território e que possam levar à disseminação internacional de doenças ${ }^{(23)}$.

O Instrumento de Decisão (Figura $1^{(24)}$ ) trazido pelo RSI 2005 estabelece uma metodologia focada nos critérios de avaliação do risco, incluindo a gravidade do impacto na saúde pública e a probabilidade de disseminação internacional, para determinar se eventos em saúde, incluindo aqueles de causa ou fonte desconhecida, constituem uma ESPII, necessitando assim de notificação formal à $O M S^{(25)}$.

Apesar de o RSI 2005 não restringir a notificação vinculando-a a uma lista pré-determinada de doenças, o Instrumento de Decisão traz duas listas de doenças: a primeira inclui varíola, poliomielite, influenza humana causada por novos subtipos e SARS, para as quais um único caso já é considerado uma ESPII e deve ser imediatamente notificado à OMS; a segunda lista inclui cólera, peste, febre amarela, febres hemorrágicas de origem viral, febre do Nilo e outras doenças de especial preocupação nacional ou regional, como dengue, febre do Rift Valley e doença meningocócica, para as quais um evento deve sempre ser avaliado com auxílio do Instrumento de Decisão, uma vez que essas doenças apresentam a habilidade de causar um sério impacto na saúde pública, bem como possuem potencial para uma rápida disseminação internacional(26).

Entretanto, a versão revisada do regulamento precisava ser flexível o suficiente para se estender além de uma lista pré-determinada de doenças, de forma a se adequar ao mundo moderno, uma vez que novos tipos de ameaças estão constantemente sendo descobertos ${ }^{(27)}$. Além de expandir o rol das doenças contempladas, o RSI 2005 também ampliou sua abrangência de modo a incluir não somente doenças transmissíveis. Pela própria definição de uma ESPII, entende-se que a ocorrência natural, a liberação acidental e o uso deliberado de agentes químicos e biológicos e de material nuclear estariam contemplados

(22) SUíçA. Artigo 6.1 do Regulamento Sanitário Internacional de 2005. Altera a antiga versão do regulamento. WORLD HEALTH ORGANIZATION. International Health Regulations (2005). 2. ed. 2008, cit. (23) SUíÇA. Artigo 9.2 do Regulamento Sanitário Internacional de 2005. Altera a antiga versão do regulamento. WORLD HEALTH ORGANIZATION. International Health Regulations (2005). 2. ed. 2008, cit. (24) SUíçA. Anexo II do Regulamento Sanitário Internacional de 2005. Altera a antiga versão do regulamento. WORLD HEALTH ORGANIZATION. International Health Regulations (2005). 2. ed. 2008, cit. (25) BAKER, Michael G.; FIDLER, David P. Global public health surveillance under new international health regulations. Emerging Infectious Diseases, Atlanta GA, v. 12, n. 7, p. 1058-1065, Jul. 2006.

(26) SUíçA. Anexo II do Regulamento Sanitário Internacional de 2005. Altera a antiga versão do regulamento. WORLD HEALTH ORGANIZATION. International Health Regulations (2005). 2. ed. 2008, cit. (27) WILSON, Kumanan; MCDOUGALL, Christopher; FORSTER, Alan. The responsibility of healthcare Institutions to Protect Global Health Security. Healthcare Quarterly, Toronto ON, v. 12, n. 1, p. 56-60, 2009. 
pelo regulamento, uma vez que podem afetar a saúde humana e representam uma potencial ameaça à saúde pública internacional|(28).

Figura 1 - Adaptação do Instrumento de Decisão do RSI 2005

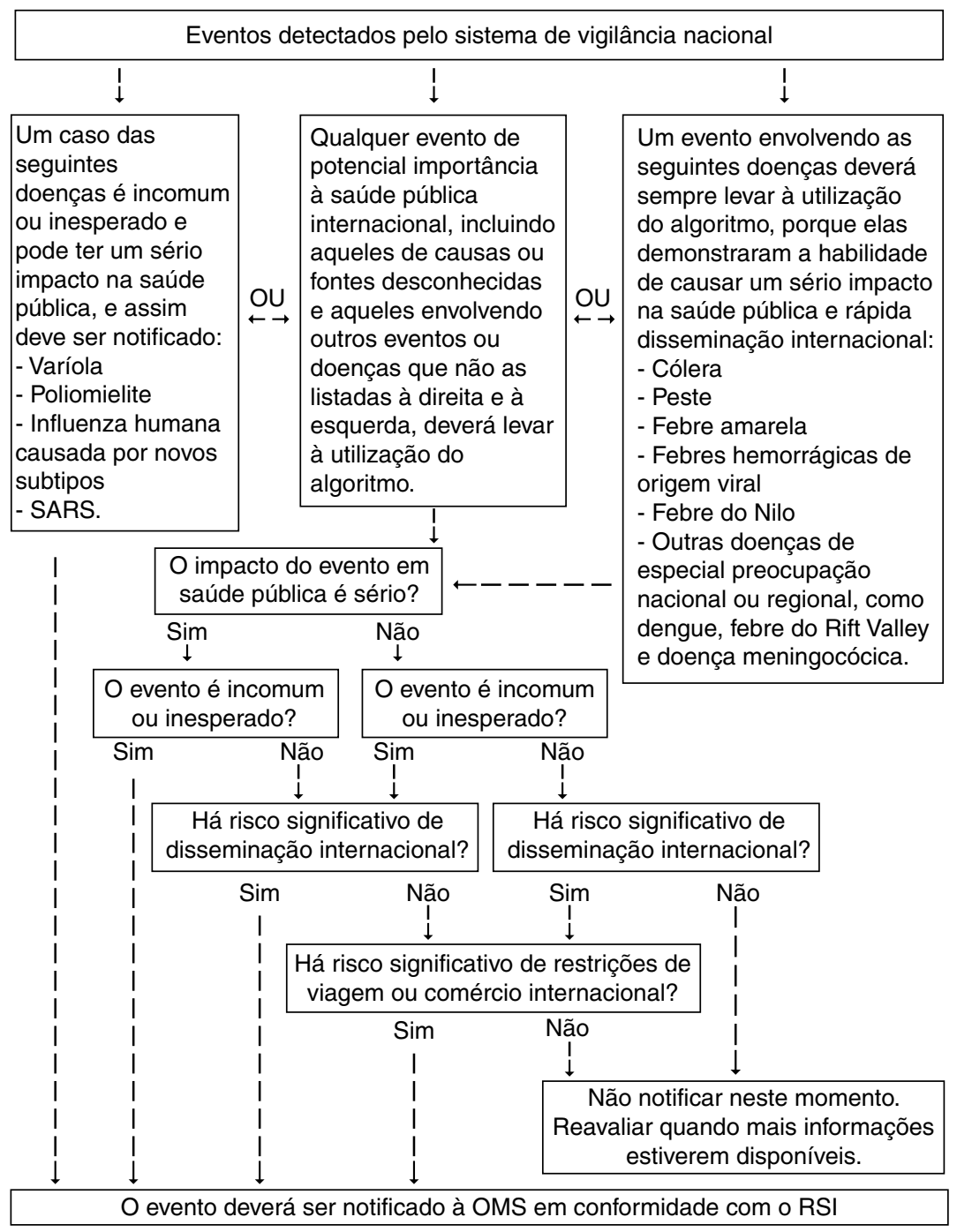

(28) SUíçA. Anexo II do Regulamento Sanitário Internacional de 2005. Altera a antiga versão do regulamento. WORLD HEALTH ORGANIZATION. International Health Regulations (2005). 2. ed. 2008, cit. 
Diante do contínuo e rápido crescimento e globalização das indústrias químicas, incidentes constituem riscos à saúde, podendo levar à ocorrência de uma ESPII, uma vez que seu impacto pode transcender o local da ocorrência original(29). Esses incidentes incluem desde uma óbvia liberação química, como um vazamento, até um evento de percepção menos imediata, como a contaminação de um produto ${ }^{(30)}$.

Da mesma forma que os agentes químicos podem ocasionar incidentes químicos, os agentes biológicos podem ocasionar incidentes biológicos, os quais constituem um potencial risco à saúde pública internacional, sendo assim uma possível ESPII. O risco deste tipo de incidente é relativamente comum, principalmente na rotina de profissionais de saúde e pacientes hospitalares, que estão em constante contato com materiais biológicos potencialmente contaminados.

Assim como os incidentes químicos e biológicos, acidentes radionucleares também constituem uma possível ESPII, uma vez que implicam um potencial risco à saúde pública, não se limitando ao local da ocorrência original, como foi o caso do acidente nuclear de Chernobyl, que ocorreu em 1986 na Ucrânia e cujo impacto alcançou também a Bielorússia e a Rússia, numa área total de 150 mil quilômetros quadrados, o que resultou na realocação de mais de 200 mil pessoas, sendo considerado por isso o pior acidente da história envolvendo energia nuclear ${ }^{(31)}$.

Mais recentemente, um terremoto de magnitude 9 ocorrido na costa leste do Japão em 11 de março de 2011 e o tsunami dele decorrente causaram um desastre radiológico comparável, senão superável, ao de Chernobyl. Apesar do desligamento automático da energia das plantas nucleares na área afetada, em virtude da detecção do terremoto, as ondas do tsunami foram as responsáveis pelos danos, principalmente em Fukushima Dai-ichi, cuja estrutura estava preparada para suportar ondas de até 5,7 m de altura; as ondas, estimadas em 14 metros, e os escombros danificaram os instrumentos e os sistemas de controle, bem como as linhas de comunicação, o que levou a uma série de incêndios no local e à disseminação da contaminação radiológica(32). De acordo com a

(29) OLOWOKURE, B.; POORANSINGH, S.; TEMPOWSKI, J.; PALMER, S.; MEREDITH, T. Global surveillance for chemical incidents of international public health concern. Bulletin of the World Health Organization, v. 83, n. 12, p. 928-934, Dec. 2005.

(30) BRITO, Maria Cecília Martins. Vigilância sanitária e proteção da saúde In: FUNDAÇÃO OSWALDO CRUZ. MINISTÉRIO DA SAÚDE. Ações de Vigilância no SUS. Rio de Janeiro, set. 2008. Disponível em: <http://www4.ensp.fiocruz.br/biblioteca/dados/txt_893718454.ppt>. Acesso em: 13 dez. 2009.

(31) INTERNATIONAL ATOMIC ENERGY AGENCY, 1957. Frequently Asked Chernobyl Questions. Disponível em: <http://www.iaea.org/NewsCenter/Features/Chernobyl-15/cherno-faq.shtml>. Acesso em: 13 dez. 2009. Perguntas freqüentes sobre Chernobyl.

(32) INTERNATIONAL ATOMIC ENERGY AGENCY, 1957. Mission Report. Disponível em: <http:// www-pub.iaea.org/mtcd/meetings/pdfplus/2011/cn200/documentation/cn200_final-fukushimamission_report.pdf>. Acesso em: 02 ago. 2012. Relatório sobre o terremoto, tsunami e desastre radiológico ocorrido em Fukushima. 
Agência Internacional de Energia Atômica (AIEA), tratou-se de um evento de nível 7, o maior possível; diante disso, o governo japonês ordenou a evacuação da área, inicialmente num raio de 20 quilômetros, que posteriormente foi aumentado para 30-50 quilômetros, em face da contínua e atípica expansão do material radioativo(33).

Além das ocorrências naturais e das liberações acidentais, é preciso ter em mente que o bioterrorismo, traduzido no uso deliberado de produtos com intuito de causar dano a uma determinada população, é uma realidade, e ações precisam ser tomadas no sentido de evitar, ou ao menos minimizar, sua ocorrência e a disseminação desses agentes. Recentes ataques bioterroristas, como os de antraz nos Estados Unidos em 2001, confirmam que uma importante parte do preparo para lidar com esse tipo de evento é o desenvolvimento de sistemas de vigilância capazes de rapidamente detectar e monitorar o curso de surtos, minimizando assim a morbidade e a mortalidade com eles associadas ${ }^{(34)}$.

Apesar de o RSI 2005 não incluir um artigo específico sobre a liberação intencional de armas de destruição em massa (ADM), a semelhança entre as obrigações estabelecidas pelos arts. 6 e 7 permite-nos inferir que o regulamento também é aplicável nesse tipo de situação. Se por um lado o regulamento precisava criar o aparato legal para permitir à OMS auxiliar na resposta aos riscos reais à saúde pública, por outro, precisava abster-se de envolvê-la nas políticas nacionais e internacionais de controle de ADM. De forma a atingir esse objetivo, o novo regulamento limita a atuação da OMS a situações de saúde pública relacionadas com a suspeita da liberação intencional de ADM, sem colocar essa entidade numa posição em que precise determinar se o Estado-Membro violou alguma de suas obrigações, conforme determinadas pelos tratados de controle de armas ou pelas resoluções do Conselho de Segurança das Nações Unidas referentes à $\mathrm{ADM}^{(35)}$.

Apesar da ampliação dos eventos abrangidos pelo RSI 2005, com a inclusão da ocorrência natural, da liberação acidental e do uso deliberado de agentes químicos e biológicos e de material nuclear, o principal foco do regulamento continua sendo a disseminação internacional das doenças transmissíveis, como a influenza. Em março de 2009, iniciou-se o surto de

(33) SUGIMOTO, A.; KRULL, S.; NOMURA, S.; MORITA, T.; TSUBOKURA, M. The voice of the most vulnerable: lessons from the nuclear crisis in Fukushima, Japan. Bulletin of the World Health Organization, n. 90, p. 629-630, 2012. Disponível em: <http://www.who.int/bulletin/ volumes/90/8/11-094474/en/>. Acesso em: 02 ago. 2012.

(34) BRAVATA, D. M.; MCDONALD, K. M.; SMITH, W. M.; RYDZAK, C.; SZETO, H.; BUCKERIDGE, D. L.; HABERLAND, C. OWENS, D. Systematic review: surveillance systems for early detection of bioterrorism-related diseases. Annals of Internal Medicine, Philadelphia PA, v. 140, n. 11, p. 910-922, Jun. 2004.

(35) FIDLER, David P. From international sanitary conventions to global health security: the new international health regulations. Chinese Journal of International Law, Oxford, v. 4, n. 2, p. 325392, 2005. 
Influenza A (H1N1) em Veracruz, México, e no mês seguinte, em 12 de abril, o diretor geral de Epidemiologia do México notificou o surto à Organização Pan-Americana da Saúde (OPAS), em conformidade com o Regulamento Sanitário Internacional; em 23 de abril, vários casos de Influenza A (H1N1) de origem suína confirmados em laboratório foram comunicados ${ }^{(36)}$. Com o crescente aumento do número de casos, a emergência da nova linhagem do vírus influenza foi elevada a uma pandemia em 11 de junho de 2009, através de uma declaração à imprensa feita pela diretora-geral da Organização Mundial da Saúde, Margaret Chan, momento em que havia aproximadamente 30 mil casos confirmados em 74 países $^{(37)}$. Essa foi a primeira ESPII a ocorrer após a implementação em 2007 da versão revisada do RSI e a avaliação da resposta global ao ocorrido servirá como base para verificar a eficácia da regulamentação em minimizar ou mesmo impedir a disseminação global da doença, de forma a melhorar o preparo das autoridades competentes para lidar com pandemias e ESPII no futuro ${ }^{(38)}$.

\section{A ESTRUTURAÇÃO DO SISTEMA DE IDENTIFICAÇÃO E NOTIFICAÇÃO DE EVENTOS}

De acordo com o RSI 2005, cabe a cada Estado-Membro estruturar um sistema de identificação e notificação de eventos, em conformidade com as responsabilidades atribuídas pelo próprio regulamento. Ao mesmo tempo em que especifica as responsabilidades de vigilância, resposta e pontos de entrada, o RSI 2005 determina as capacidades que cada país deverá desenvolver para ser capaz de cumprir com suas respectivas atividades ${ }^{(39)}$.

Com esse objetivo, o estabelecimento de um Ponto Focal Nacional para o RSI e a designação das autoridades responsáveis e suas respectivas áreas de jurisdição são responsabilidades imputadas aos Estados-Membros pelo novo

(36) CENTERS FOR DISEASE CONTROL AND PREVENTION (CDC). Outbreak of Swine-Origin Influenza A (H1N1) Virus Infection --- Mexico, Mar.-Apr. 2009. Disponível em: <http://www.cdc.gov/ $\mathrm{mmwr} /$ preview/mmwrhtml/mm58d0430a2.htm>. Acesso em: 30 jul. 2012.

(37) WORLD HEALTH ORGANIZATION. World now at the start of 2009 influenza pandemic. 2009. Disponível em: <http://www.who.int/mediacentre/news/statements/2009/h1n1_pandemic _ phase6_20090611/en/index.html>. Acesso em: 30 jul. 2012.

(38) Id. How will the global response to the pandemic H1N1 be reviewed? Apr. 1948. Disponível em: $\quad$ http://www.who.int/csr/disease/swineflu/frequently_asked_questions/review_committee/ en/index.html>. Acesso em: 30 jul. 2012. Apresenta dados sobre a revisão da resposta global à pandemia de $\mathrm{H} 1 \mathrm{~N} 1$.

(39) BAKER, Michael. G.; FORSYTH, Andrew M. The new international health regulations: a revolutionary change in global health security. The New Zealand Medical Journal, Nova Zelândia, v. 120, n. 1267, p. 1-8, Dec. 2007. 
regulamento ${ }^{(40)}$. Já em 2005, logo após a publicação do novo regulamento, o Ministério da Saúde designou a Secretaria de Vigilância em Saúde (SVS) como o Ponto Focal Nacional para o RSI. Dessa forma, a SVS é o canal de comunicação externa do Brasil para todos os assuntos referentes ao RSI $2005^{(41)}$.

Apesar de a coleta de dados não ser responsabilidade direta da SVS, a transmissão das informações para a OMS é. Ou seja, é responsabilidade da SVS enviar à OMS, sempre que necessário e seguindo a cadeia de comunicação estabelecida pelo regulamento, comunicações relativas a uma ESPII(42).

Além disso, também cabe à SVS colocar em prática o novo regulamento e disseminar as informações aos órgãos e setores brasileiros conforme a necessidade, bem como consolidar as informações deles provenientes, incluindo notificações e dados oriundos de postos de fronteiras, serviços de saúde pública, clínicas e hospitais ${ }^{(43)}$.

A cadeia de comunicação não liga a SVS diretamente à OMS. Essa comunicação é intermediada pelo Ponto de Contato da OMS, para o qual a SVS deve estar permanentemente acessível. No caso do Brasil, o intermédio é feito pela OPAS, por meio do Centro de Operações de Urgência ${ }^{(44)}$.

A transmissão da informação, desde a identificação de eventos até a comunicação à OMS, passando pela SVS e pela OPAS, constitui a cadeia de comunicação, uma capacidade fundamental prevista pelo RSI 2005 para o processo de vigilância em saúde que garante a detecção, a avaliação e a notificação de eventos, desde o nível local até o internacional (Figura 2).

O recebimento e o processamento das notificações são feitos em nível nacional pelo Centro de Informações Estratégicas em Vigilância em Saúde (Cievs) do Ministério da Saúde, criado pela Portaria $n^{\circ} 30^{(45)}$ e inaugurado em março de 2006. Ele consiste numa equipe especializada responsável por receber as notificações de surtos e emergências epidemiológicas que coloquem em risco a saúde

(40) SUÍÇA. Artigo 4 do Regulamento Sanitário Internacional de 2005. Altera a antiga versão do regulamento. WORLD HEALTH ORGANIZATION. International Health Regulations (2005). 2. ed. 2008. Disponível em: <http://whqlibdoc.who.int/publications/2008/9789241580410_eng.pdf>. Acesso em: 13 dez. 2009.

(41) NOVO Regulamento Sanitário entra em Vigor. Boletim Eletrônico da Secretaria de Vigilância em Saúde, n. 29, maio 2007. Disponível em: <http://portal.saude.gov.br/portal/arquivos/pdf/svs_maio_b. pdf>. Acesso em: 13 dez. 2009.

(42) Id. Ibid.

(43) Id. Ibid.

(44) ORGANIZAÇÃO PAN-AMERICANA DA SAÚDE, 1902. Relatório da $27^{a}$ Conferência Sanitária Pan-Americana. Disponível em: <http://www.paho.org/Portuguese/GOV/CSP/csp27-fr-p.pdf>. Acesso em: 13 dez. 2009.

(45) MINISTÉRIO DA SAÚDE. Portaria n. 30, de 7 de julho de 2005. "Institui o Centro de Informações Estratégicas em Vigilância em Saúde, define suas atribuições, composição e coordenação". Disponível em: <http://portal.saude.gov.br/portal/arquivos/pdf/do1-61_Institui\%20 CIEVS.pdf>. Acesso em: 15 out. 2012. 
da população em qualquer local do país e comunicar às autoridades competentes sempre que necessário. Essa equipe trabalha 24 horas por dia, todos os dias da semana, evitando assim demora na comunicação de uma emergência, e dispõe de recursos tecnológicos de última geração para a realização de suas funções, incluindo televisores, computadores, aparelhos de videoconferência, servidores de dados, telefones via satélites, telefones com palmtop e placas de conexão via celular ${ }^{(46)}$.

Além de fornecer suporte em situações de crise, o Cievs também exerce papel fundamental na coordenação do sistema nacional de vigilância em saúde,

Figura 2 - Cadeia de comunicação do sistema de vigilância em saúde pública(47)

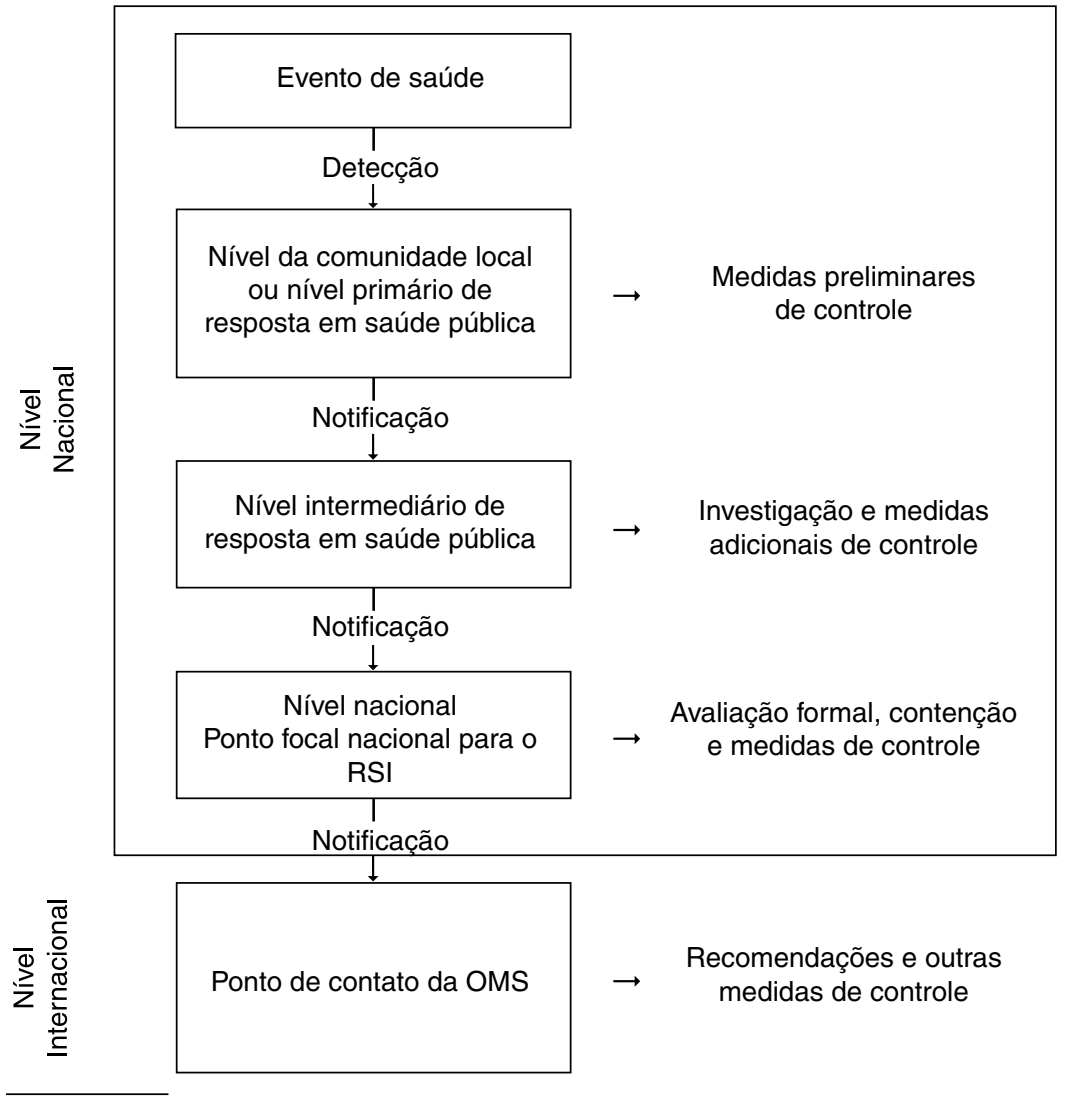

(46) MINISTÉRIO DA SAÚDE. Centro de Informações Estratégicas em Vigilância em Saúde - CIEVS. Disponível em: <http://portal.saude.gov.br/portal/saude/profissional/visualizar_texto.cfm?idtxt=32296> . Acesso em: 13 dez. 2009.

(47) BAKER, M.G.; FILDER, D.P. op.cit., p. 1058-1065. 
no acompanhamento do padrão das doenças de elevado potencial de disseminação e/ou riscos à saúde pública e na identificação de doenças de origem desconhecida ou alteração do padrão epidemiológico de doenças conhecidas. O Cievs não apenas analisa as notificações recebidas como também avalia diariamente os principais meios de comunicação do país, a fim de sempre avaliar os eventos em saúde e detectar quais são de relevância para realização de uma investigação epidemiológica e aplicação das medidas cabíveis ${ }^{(48)}$.

\section{CONSIDERAÇÕES FINAIS}

O RSI e sua mais recente revisão representam um importante marco na legislação sanitária internacional. Além de acrescer as possíveis fontes que a OMS pode usar para identificar uma ESPII, o novo regulamento também aumentou os eventos abrangidos, incluindo ainda a ocorrência natural, a liberação acidental e o uso deliberado de agentes químicos e biológicos e de material nuclear, além das doenças transmissíveis já presentes na versão anterior.

Essa ampliação das possíveis fontes de informação concedeu maior liberdade à OMS, uma vez que ela pode consultar os diversos sistemas de vigilância existentes e mantidos por países ou mesmo por entidades particulares, bem como coletar as informações que julgar pertinentes à identificação de uma ESPII. Dessa forma, evita-se que os países ignorem ou escondam informações sobre surtos que ocorrem em seu território, contribuindo assim para a internacionalização do sistema de vigilância em saúde.

Além disso, o RSI 2005 estabelece uma cadeia de comunicação para notificação de uma ESPII, especificando as responsabilidades e capacidades que cada Estado-Membro deve desenvolver para garantir a detecção e a avaliação de eventos em saúde, bem como sua notificação desde o nível local até o internacional. Todavia, o instrumento é omisso ao não abarcar nenhuma estipulação efetiva que possa abrandar as consternações dos indivíduos, comunidades ou até mesmo países.

O RSI 2005 se mostra promissor para minimizar a propagação internacional de enfermidades e prever comunicações de surtos epidêmicos e outros riscos, pois a atual versão do instrumento é mais abrangente. Entretanto, o aprimoramento da segurança sanitária envolve vários fatores e níveis de abrangência para ser realmente concretizado, e a busca pela segurança sanitária necessita também da reestruturação de instituições, tanto no aspecto técnico como na administração por parte dos Estados-Membros.

(48) MINISTÉRIO DA SAÚDE. Centro de Informações Estratégicas em Vigilância em Saúde CIEVS, cit. 
Outro importante ponto que deve ser levado em consideração é quanto os Estados-Membros da OMS são diferentes entre si, desde os recursos técnicos e econômicos a seu dispor até diferenças políticas, culturais e religiosas.

Países pobres, imersos em inúmeras dificuldades, não só na área da saúde, que não contam com o mínimo de infraestrutura básica para atender satisfatoriamente situações menos complexas que a transnacionalização dos riscos, jamais conseguirão atender às capacidades nacionais básicas para detectar, avaliar, notificar e informar uma ESPII prevista no RSI sem ajuda técnica e financeira, que será realmente eficiente apenas se for realizada de modo estruturante e condizente com suas reais necessidades. O novo instrumento regulatório carece da previsão expressa de um plano ou um fundo, com recursos destinados a ajudar as nações mais pobres a estruturarem instituições imprescindíveis para cumprir o RSI, tendo em vista que, para elas, há previsão de muitas obrigações e de pouca cooperação efetiva por parte dos países mais ricos.

A visão de que se pode controlar totalmente a ocorrência e o alastramento de eventos em saúde é demasiadamente otimista. Mesmo com a regulação de viajantes, aeronaves e cargas, fronteiras sempre serão permeáveis, por exemplo, em razão de doenças com longo período de incubação e eventos da natureza. Sempre que possível, o intuito do regulamento é evitar a ocorrência de eventos em saúde; porém, quando não consegue atingir essa proposição, o objetivo torna-se minimizar o alastramento e diminuir a gravidade das consequências.

Para angariar o mínimo de segurança faz-se necessária vontade política concisa, comprometida com a reestruturação do aparato sanitário em âmbito internacional, nacional e regional. A identificação, notificação e resposta a eventos sanitários que podem apresentar riscos internacionais serão mais eficazes na medida em que se disponha de infraestrutura básica adequada, sendo fundamental o investimento em longo prazo e uma governança em saúde.

\section{REFERÊNCIAS}

AGÊNCIA NACIONAL DE VIGILÂNCIA SANITÁRIA. Notícias da Anvisa.Leite: Anvisa acompanha Operação Ouro Branco. Brasília, 23 out. 2007. Disponível em: <http://www.anvisa.gov.br/DIVULGA/noticias/2007/231007_2.htm>. Acesso em: 13 dez. 2009.

BAKER, Michael G.; FIDLER, David P. Global public health surveillance under new international health regulations. Emerging Infectious Diseases, Atlanta GA, v. 12, n. 7, p. 1058-1065, Jul. 2006.

FORSYTH, Andrew M. The new international health regulations: a revolutionary change in global health security. The New Zealand Medical Journal, Nova Zelândia, v. 120, n. 1267, p. 1-8, Dec. 2007. 
BERLINGUER, Giovanni. Globalização e saúde global. Estudos Avançados, São Paulo, v. 13, n. 35, p. 21-38, 1999.

BRAVATA, D. M.; MCDONALD, K. M.; SMITH, W. M.; RYDZAK, C.; SZETO, H.; BUCKERIDGE, D. L.; HABERLAND, C. OWENS, D. Systematic review: surveillance systems for early detection of bioterrorism-related diseases. Annals of Internal Medicine, Philadelphia PA, v. 140, n. 11, p. 910-922, Jun. 2004.

BRITO, Maria Cristina Martins. Vigilância sanitária e proteção da saúde In: FUNDAÇÃO OSWALDO CRUZ. MINISTÉRIO DA SAÚDE. Ações de Vigilância no SUS. Rio de Janeiro, set. 2008. Disponível em: <http://www4.ensp.fiocruz.br/ biblioteca/dados/txt_893718454.ppt>. Acesso em: 13 dez. 2009.

BROWNSTEIN, John S.; FREIFELD, Clark C. HealthMap: the development of automated real-time internet surveillance for epidemic intelligence. Eurosurveillance, Suíça, v. 12, n. 48, pii =3322, Nov. 2007. Disponível em: $<$ http://www.eurosurveillance.org/ViewArticle.aspx?Articleld=3322>. Acesso em: 13 dez. 2009.

CENTERS FOR DISEASE CONTROL AND PREVENTION (CDC). Outbreak of Swine-Origin Influenza A (H1N1) Virus Infection --- Mexico, Mar.-Apr. 2009. Disponível em: <http://www.cdc.gov/mmwr/preview/mmwrhtml/mm58d0430a2. htm>. Acesso em: 30 jul. 2012.

FIDLER, David P. From international sanitary conventions to global health security: the new international health regulations. Chinese Journal of International Law, Oxford, v. 4, n. 2, p. 325-392, 2005.

Globalization, international law, and emerging infectious diseases. Emerging Infectious Diseases, v. 2, n. 2, 1996.

GONZALO, Miguel Mínguez. The new international health regulations (2005 IHR). Revista Española de Salud Pública, Madrid, v. 81, n. 3, p. 239-246, maio/ jun. 2007.

HEALTHMAP, 2007. Global Disease Alert Map. Disponível em: <http://www. healthmap.org/about.php>. Acesso em: 13 dez. 2009.

HEYMANN, David L. SARS and emerging infectious diseases: a challenge to place global solidarity above national sovereignty. Annals Academy of Medicine, Singapura, v. 35, n. 5, p. 350-353, 2006.

INTERNATIONAL ATOMIC ENERGY AGENCY, 1957. Frequently Asked Chernobyl Questions. Disponível em: <http://www.iaea.org/NewsCenter/ Features/Chernobyl-15/cherno-faq.shtml>. Acesso em: 13 dez. 2009.

Mission Report. Disponível em: <http://www-pub.iaea.org/mtcd/ meetings/pdfplus/2011/cn200/documentation/cn200_final-fukushima-mission_ report.pdf>. Acesso em: 02 ago. 2012. 
INTERNATIONAL SOCIETY FOR INFECTIOUS DISEASES. Program for Monitoring Emerging Diseases - ProMed-mail. 1986. Disponível em: <http:// www.promedmail.org/pls/otn/f?p=2400:1950:2415474060216450::NO:::>. Acesso em: 13 dez. 2009.

KATZ, Rebecca. Use of Revised International Health Regulations During Influenza A (H1N1) Epidemic. Emerging Infectious Diseases, Atlanta GA, v. 15, n. 8, p. 1165-1170, ago. 2009.

MERIANOS, Angela; PEIRIS, Malik. International Health Regulations. The Lancet, New York NY, v. 366, n. 9493, p. 1249-1251, Oct. 2005.

MINISTÉRIO DA SAÚDE. Centro de Informações Estratégicas em Vigilância em Saúde - CIEVS. Disponível em: <http://portal.saude.gov.br/portal/saude/ profissional/visualizar_texto.cfm?idtxt=32296>. Acesso em: 13 dez. 2009.

NOVO Regulamento Sanitário entra em Vigor. Boletim Eletrônico da Secretaria de Vigilância em Saúde, n. 29, maio 2007. Disponível em: <http://portal.saude.gov. br/portal/arquivos/pdf/svs_maio_b.pdf>. Acesso em: 13 dez. 2009.

OLOWOKURE, B.; POORANSINGH, S.; TEMPOWSKI, J.; PALMER, S.; MEREDITH, T. Global surveillance for chemical incidents of international public health concern. Bulletin of the World Health Organization, v. 83, n. 12, p. 928934, Dec. 2005.

ORGANIZAÇÃO PAN-AMERICANA DA SAÚDE, 1902. Relatório da $27^{\mathrm{a}}$ Conferência Sanitária Pan-Americana. Disponível em: <http://www.paho.org/ Portuguese/GOV/CSP/csp27-fr-p.pdf>. Acesso em: 13 dez. 2009.

ORGANIZACIÓN MUNDIAL DE LA SALUD. Evolución de la seguridad sanitaria. In: INFORME sobre la salud en el mundo 2007 - Un porvenir más seguro: protección de la salud pública mundial en el siglo XXI. Geneva, 2007. p. 1-15. Disponível em: <http://www.who.int/whr/2007/07_report_es.pdf>. Acesso em: 13 dez. 2009.

PUBLIC HEALTH AGENCY OF CANADA. The Global Public Health Intelligence Network (GPHIN), Set. 2004. Disponível em: <http://www.phac-aspc.gc.ca/media/ nr-rp/2004/2004_gphin-rmispbk-eng.php>. Acesso em: 13 dez. 2009.

RODIER, Guénaël; HARDIMAN, Max; PLOTKIN, B.; GANTER, B. Implementing the International Health Regulations (2005). Europe Eurosurveillance, Suíça, v. 11, n. 12, pii=664, Dec. 2006. Disponível em: <http://www.eurosurveillance.org/ ViewArticle. aspx?Articleld=664>. Acesso em: 13 dez. 2009.

SUGIMOTO, A. KRULL, S.; NOMURA, S.; MORITA, T.; TSUBOKURA, M. The voice of the most vulnerable: lessons from the nuclear crisis in Fukushima, Japan. Bulletin of the World Health Organization, n. 90, p. 629-630, 2012. Disponível em: <http://www.who.int/bulletin/volumes/90/8/11-094474/en/>. Acesso em: 02 ago. 2012. 
TUCKER, Jonathan B. Updating the international health regulations. Biosecurity and Bioterrorism: biodefense strategy, practice, and science, New Rochelle NY, v. 3, n. 4, p. 338-347, 2005.

VíTIMAS da hemodiálise ao Deus dará. Ministério Público de Pernambuco, Pernambuco, 20 fev. 2008. Disponível em: <http://www.mp.pe.gov.br/index.pl/ clipagem2002_vitimas>. Acesso em: 13 dez. 2009.

WILSON, Kumanan; MCDOUGALL, Christopher; FORSTER, Alan. The responsibility of healthcare Institutions to Protect Global Health Security. Healthcare Quarterly, Toronto ON, v. 12, n. 1, p. 56-60, 2009.

WORLD HEALTH ORGANIZATION. Constitución de la Organización Mundial de la Salud. Disponível em: <http://www.who.int/governance/eb/who_constitution_ sp.pdf>. Acesso em: 14 ago. 2012.

Global Outbreak Alert and Response Network - GOARN. Apr. 1948. Disponível em: <http://www.who.int/csr/outbreaknetwork/en>. Acesso em: 13 dez. 2009.

How will the global response to the pandemic H1N1 be reviewed? Apr. 1948. Disponível em: <http://www.who.int/csr/disease/swineflu/frequently_ asked_questions/review_committee/en/index.html>. Acesso em: 30 jul. 2012.

International Health Regulations (1969). 3. ed. Disponível em: <http:// www.who.int/csr/ihr/ihr1969.pdf>. Acesso em: 13 dez. 2009.

International Health Regulations (2005). 2. ed. 2008. Disponível em: <http://whqlibdoc.who.int/publications/2008/9789241580410_eng.pdf>. Acesso em: 13 dez. 2009.

WHO definition of Health. Apr. 1948. Disponível em: <http://www.who. int/about/definition/en/print.html>. Acesso em: 13 dez. 2009.

World now at the start of 2009 influenza pandemic. 2009. Disponível em: <http://www.who.int/mediacentre/news/statements/2009/h1n1_pandemic_ phase6_20090611/en/index.html>. Acesso em: 30 jul. 2012. 